\title{
Frontal Alpha Asymmetry Correlates with Suicidal Behavior in Major Depressive Disorder
}

\author{
Yeonsoo Park ${ }^{1, *}$, Wookyoung Jung ${ }^{2, *}$, Sungkean Kim ${ }^{1,3}$, Hyunjin Jeon ${ }^{1}$, Seung-Hwan Lee ${ }^{1,4}$ \\ ${ }^{1}$ Clinical Emotion and Cognition Research Laboratory, Department of Psychiatry, Inje University, Goyang, ${ }^{2}$ Department of Psychology, Keimyung \\ University, Daegu, ${ }^{3}$ Department of Biomedical Engineering, Hanyang University, Seoul, ${ }^{4}$ Department of Psychiatry, Inje University Ilsan Paik \\ Hospital, Inje University College of Medicine, Goyang, Korea
}

\begin{abstract}
Objective: Based on the constant associations made between major depressive disorder (MDD) and alpha asymmetry, and MDD and suicide, this study aimed to examine the relationship between frontal alpha asymmetry and suicide in MDD patients.

Methods: Sixty-six MDD patients, of whom fifteen were male and fifty-one were female, were recruited. Independent groups were created based on the median score of frontal alpha asymmetry: the left dominant (LD) group and the right dominant $(\mathrm{RD})$ group. The alpha band $\left(8^{-12} \mathrm{~Hz}\right)$ and its sub-bands (i.e., low alpha band: $8-10 \mathrm{~Hz}$; high alpha band: 10-12 Hz) were of interest. Source level alpha asymmetry was calculated as well.

Results: Suicidal behavior was positively correlated with the asymmetry indices of the low alpha band and the alpha band in the LD group and that of the high alpha band in the RD group. Source level analysis revealed positive correlations between suicidal behavior and the asymmetry index of the low alpha band in the LD group.

Conclusion: Frontal alpha asymmetry, especially that of the low alpha band, might reflect the cognitive deficits associated with suicidal behaviors in MDD patients.
\end{abstract}

KEY WORDS: Alpha asymmetry; Depression; Suicide; Electroencephalography.

\section{INTRODUCTION}

Among the various psychiatric disorders that have been associated with suicidal behavior, depression has been one of the most consistent and robust predictors of suicide. ${ }^{1,2)}$ Even after controlling the effects of other comorbid disorders, depression is still a significant predictor of suicidal behavior, especially suicide ideation. ${ }^{3)}$ It is thus crucial to assess suicide risk when treating patients with depression.

In general, abnormal alpha band activity has been suggested as an indicator of depression. ${ }^{4,5)}$ Perhaps, one of the most promising biomarkers of depression might be

Received: April 11, 2018/ Revised: May 30, 2018

Accepted: June 28, 2018

Address for correspondence: Seung-Hwan Lee

Department of Psychiatry, Inje University Ilsan Paik Hospital, Inje

University College of Medicine, 170 Juhwa-ro, Ilsanseo-gu,

Goyang 10380, Korea

E-mail: Ishpss@paik.ac.kr

ORCID: https://orcid.org/0000-0003-0305-3709

*These authors contributed equally to this work. asymmetrical electroencephalographic (EEG) activity during the resting state. Characterized by asymmetrical alpha band activity $(8-12 \mathrm{~Hz})$ between the left and the right frontal regions (i.e., alpha asymmetry), ${ }^{6}$ ) alpha asymmetry distinguishes individuals with depression from individuals without depression. ${ }^{7-9)}$ In particular, depressive individuals typically display a greater alpha activity in their left hemisphere than in their right hemisphere. The enhanced alpha power is thought to reflect a reduction in cortical activity in the corresponding region, and hypoactivation in the left frontal hemisphere has been reported in individuals with depression. ${ }^{10-12)}$

In addition, frontal alpha asymmetry has been examined in relations to specific cortical regions including the dorsolateral prefrontal cortex (DLPFC), the ventromedial prefrontal cortex (vmPFC), the anterior cingulate cortex (ACC), the rostral ACC (rACC), and the subgenual ACC (sgACC), has been extensively examined. ${ }^{13-16)}$ Impairments in the DLPFC and the vmPFC have been associated with deficits in negative emotional control and automated

(ㄷ) This is an Open-Access article distributed under the terms of the Creative Commons Attribution Non-Commercial License (http://creativecommons.org/licenses/by-nc/4.0) which permits unrestricted non-commercial use, distribution, and reproduction in any medium, provided the original work is properly cited. 
processing of emotionally relevant stimuli, respectively. ${ }^{17,18)}$ Moreover, patients with major depressive disorder (MDD) have shown reduced perfusion or metabolic activity in the DLPFC, particularly in the left hemisphere, ${ }^{19-21)}$ as well as hypoperfusion or hypometabolism in the ACC, which is thought to be a byproduct of reduced cortical volume of the left sgACC. ${ }^{22-24)}$ Furthermore, disturbed functioning as well as reduced volumes of the orbitofrontal cortex (OFC) have been reported to be associated with increased impulsivity and aggression, which are known as risk factors of suicidal behaviors. ${ }^{23,25-28)}$

Despite the strong evidence supporting the relationships between alpha asymmetry and depression, and depression and suicide, the association between alpha asymmetry and suicidal behaviors has been relatively unexplored. The only study that has directly examined the relationship between alpha asymmetry and suicide was conducted in female adolescents. ${ }^{29)}$ Results indicated that depressive suicidal adolescents display a greater alpha activity in the left than the right hemisphere. Given that suicidal individuals are often characterized by cognitive rigidity and attentional biases that are associated with impaired frontal lobe functioning, ${ }^{30-33)}$ it is worthwhile to examine alpha asymmetry and suicidal behavior in adult patients with MDD.

Meanwhile, studies on EEG alpha power suggest the need to distinguish the alpha band into two sub-bands: the low alpha $\left(8^{-10} \mathrm{~Hz}\right)$ and the high alpha (10-12 $\mathrm{Hz}){ }^{34,35)}$ Low alpha is thought to represent a diffused attentional and brain-state phenomenon, whereas high alpha is thought to represent more localized and task-specific cognition, such as semantic memory. ${ }^{36)}$ In a group of ADHD patients, alpha asymmetry of the low alpha band indicated the existence of clinically significant symptoms. ${ }^{37)}$ The role of the low and high alpha bands in depression, however, remains equivocal and, to our knowledge, their relationship to suicidal behavior has not been previously examined.

The purpose of the present study was to explore the relationship between frontal alpha asymmetry and suicidal behavior. In addition, we aimed to examine how the cortical level alpha asymmetries would correlate with suicidal risk. We hypothesized that suicidal behaviors would correlate with a greater relative alpha power in the left hemisphere as compared to the right hemisphere. More specifically, we hypothesized that the low alpha band, which is thought to be associated with attention, would be related to suicidality, because suicidal individuals often display cognitive deficits. ${ }^{38,39)}$ For example, attentional bias has been discovered in suicidal individuals in that they often fixate on suicidal cues. ${ }^{40,41)}$

\section{METHODS}

\section{Participants}

Seventy-eight participants with MDD were included in the study. Among those, 12 were excluded due to an insufficient number of epochs in their EEG data. A total of 66 subjects, of whom 15 were male $(22.7 \%)$ and 51 were female $(77.3 \%)$, were included in the initial analyses. The mean age was 44.77 years (standard deviation [SD], 13.62 years; range, $19-65$ years) and the mean years of education was 12.77 years (SD, 6.41 years). When asked about prior suicide attempt, 24 participants (36.4\%) reported one or more lifetime attempts, whereas 42 (63.6\%) reported no prior history. All participants were right-handed and those with brain damage or histories of psychotic symptoms or bipolar disorder were excluded from the analyses. Although our participants were all diagnosed with MDD, some exhibited greater relative alpha power in their right hemisphere than in their left. This pattern contradicted the previous findings identified in MDD patients (i.e., greater relative alpha power in the left hemisphere).

Groups were created based on the median scores of the asymmetry indices of the alpha bands at each region of interest (ROI): FP1-FP2 (low alpha, 1.80; high alpha, 1.13; alpha, 1.24), F3-F4 (low alpha, 2.79; high alpha, 2.49; alpha, 2.66), and F7-F8 (low alpha, 5.22; high alpha, 4.14; alpha, 4.52). All participants were matched to either the left-dominant (LD) group or the right-dominant (RD) group for all three band frequencies at each site. Only those who fell into the same group for all three band frequencies at each site were included in the final analyses ( $\mathrm{n}=56$ ). Participants signed an informed consent approved by the Institutional Review Board at Inje University Ilsan Paik Hospital (2015-03-212) prior to their participation.

As a part of their treatment, most of the participants ( $\mathrm{n}=$ 52) received antidepressants. The most common type of antidepressant in use was escitalopram followed by venlafaxine, paxil, tianeptine, and sertraline. In addition, 
most $(n=45)$ were treated with benzodiazepines such as lorazepam, clonazepam, alprazolam, and etizolam.

\section{Psychological Measures}

\section{Cognitive Flexibility Inventory (CFI)}

Cognitive flexibility was measured with the Korean version of the $\mathrm{CFI}^{42)}$ The scale consists of two subscales, the control subscale and the alternative subscale. The control subscale measures the extent to which individuals believe they are capable of controlling problematic situations; the alternative subscale measures how much individuals believe in their ability to find alternative solutions in unfavorable situations. A total of nineteen questions are measured on a 7-point Likert scale, in which higher total scores indicate greater cognitive flexibility. In the present study, the internal consistencies (Cronbach's alpha) for the alternative subscale, the control subscale, and the entire inventory were $0.87,0.90$, and 0.91 , respectively.

\section{Rumination Reflection Questionnaire (RRQ)}

Focused attention was assessed with the Korean version of the RRQ. ${ }^{43)}$ Rumination is defined as fixation on negative thoughts, whereas reflection is defined as introspection that promotes self-growth. The RRQ can measure both traits (RRQT) and states (RRQS). The Rumination and Reflection Questionnaires, each consists of 12 items that are measured on a 5-point Likert scale. In the present study, both trait and state rumination and reflection were assessed. Rumination ( $\alpha=0.82)$, reflection ( $\alpha=0.81)$ and the entire questionnaire $(\alpha=0.86)$ all exhibited high internal consistencies.

\section{Beck Depression Inventory (BDI) ॥}

Depression was assessed with the Korean version of the Beck Depression Inventory (i.e., BDI-II). The BDI-II is consisted of 21 items that are rated on a 4-point scale. The Korean version of the BDI-II has shown a high internal consistency $(\alpha=0.85)$ and test-retest reliability $(r=$ 0.75). ${ }^{44)}$ Cronbach's alpha for the current sample was 0.95 .

\section{Beck Anxiety Inventory (BAI)}

Anxiety was measured with the Korean version of the BAI, a self-reported questionnaire composed of 21 items. Its internal consistency and test-retest reliability were
0.93, and 0.84, respectively. ${ }^{45)}$ Cronbach's alpha for the current sample was 0.94 .

\section{Suicidal Behavior Questionnaire-Revised (SBQ-R)}

Suicidal behavior was measured through the Korean translation of the SBQ-R. ${ }^{46)}$ The SBQ-R is composed of 4 items that measure different dimensions of suicidality; 1 ) lifetime suicide ideation and suicide attempt, 2) the frequency of suicidal ideation over the past 12 months, 3) the threat of suicidal behavior, and 4) the likelihood of suicidal behavior by self-report. ${ }^{47)}$ The SBQ-R, which has exhibited excellent reliability, ${ }^{48)}$ was positively correlated with the Korean version of the Beck Scale for Suicide Ideation $(r=0.62) .{ }^{49)}$ The total scores of the SBQ-R range from 3 to 18. The Cronbach's alpha for the Korean version of the SBQ-R in the original study was 0.93 , and 0.85 for the current sample.

\section{EEG Recordings and Quantitative EEG Analysis}

Resting state EEG data was collected in a sound attenuated room for three minutes each during two conditions: the eyes-closed condition and the eyes-opened condition. The EEG signal was obtained using a 62 channel (FP1, FPZ, FP2, AF3, AF4, F7, F5, F3, F1, FZ, F2, F4, F6, F8, FT7, FC5, FC3, FC1, FCZ, FC2, FC4, FC6, FT8, T7, C5, C3, C1, CZ, C2, C4, C6, T8, TP7, CP5, CP3, CP1, CPZ, CP2, CP4, CP6, TP8, P7, P5, P3, P1, PZ, P2, P4, P6, P8, PO7, PO5, PO3, POZ, PO4, PO6, PO8, CB1, O1, OZ, O2, and CB2) Quik Cap and a NeuroScan SynAmps amplifier (Compumedics USA, El Paso, TX, USA). An international 10-20 placement scheme was applied. The ground electrode was located on the forehead and Cz was used as the reference electrode. We attached the vertical electrooculogram (EOG) electrodes above and below the left eye, and the horizontal EOG on the outer canthus of both eyes. The EEG data was recorded once the impedance fell below $5 \mathrm{k} \Omega$. A 0.1 to $100 \mathrm{~Hz}$ band pass filter at a sampling rate of $1,000 \mathrm{~Hz}$ was applied to process the all of the data.

SCAN 4.3 software (Advanced Medical Equipment Ltd., Horsham, UK) was used to preprocess the EEG data. An experienced technician with no prior knowledge of the origin of the data screened artifacts such as eye movements. The current study used eyes-opened data for its analyses. A total of 30 epochs of which the length of each epoch was configured as 2 seconds (2,048 points), were prepared for each participant. Epochs with signals 
exceeding $\pm 100 \mu \mathrm{V}$ on any channel were rejected from further analysis. A fast Fourier transformation (FFT) was performed on each epoch for 62 electrode channels to analyze the spectral powers. The FFT window size was set to match the length of each epoch (2,048 points) with the hamming window, and no overlap was allowed. The frequency bin size was $\sim 0.488 \mathrm{~Hz}(1,000 / 2,048)$ with a total of 1,025 bins. After FFT was conducted on the $30 \mathrm{ep}$ ochs, the resultant spectral power was averaged. Band powers of three frequency bands (i.e., low alpha: 8-10 $\mathrm{Hz}$; high alpha: 10-12 Hz; alpha: 8-12 Hz) were then calculated. ${ }^{50)}$ The closest frequencies corresponding to each band were selected to determine the bin size.

Frontal alpha asymmetry was measured on three paired sites: pre-frontal (FP1-FP2), mid-frontal (F3-F4), and lateral-frontal (F7-F8). ${ }^{51-53)}$ To measure alpha asymmetry, we compared the corresponding frequency band percen- tages of the left and right hemispheres. More specifically, the difference between the two hemispheres was divided by their sum (i.e., alpha asymmetry $=\left[P_{\text {left }}-P_{\text {right }}\right] /\left[P_{\text {left }}\right.$ $+P_{\text {right }} \times 100 ; P_{\text {left }}$ and $P_{\text {right }}$ are absolute powers). An asymmetry value of zero indicates that alpha power and brain activity are equal in both hemispheres; a positive asymmetry value indicates greater alpha power and hypoactivation of the left hemisphere; a negative asymmetry value indicates greater alpha power and hypoactivation of the right hemisphere. The asymmetry indices were calculated for the three alpha bands of interest.

\section{Source Activity Analysis}

The cortical distribution of the standardized source current density of brain sources in the resting state for the frequency domain was calculated through standardized low-resolution brain electromagnetic tomography

Table 1. Demographic information of the participants including means and standard deviations

\begin{tabular}{|c|c|c|c|c|}
\hline Variable & Participant $(n=66)$ & LD group $(n=28)$ & RD group $(n=28)$ & $p$ value \\
\hline Sex & & & & 0.001 \\
\hline Male & $15(22.7)$ & $11(39.3)$ & $1(30.6)$ & \\
\hline Female & $51(77.3)$ & $17(60.7)$ & $27(960.4)$ & \\
\hline Age (yr) & $44.77 \pm 13.62$ & $46.39 \pm 11.54$ & $40.86 \pm 15.65$ & 0.139 \\
\hline Education (yr) & $12.77 \pm 6.41$ & $13.93 \pm 9.05$ & $11.78 \pm 3.47$ & 0.255 \\
\hline Past suicide attempt & & & & 0.406 \\
\hline Yes & $24(36.4)$ & $8(28.6)$ & $11(39.3)$ & \\
\hline No & $42(63.6)$ & $20(71.4)$ & $17(60.7)$ & \\
\hline CFI & $81.36 \pm 19.04$ & $82.68 \pm 16.27$ & $80.25 \pm 20.31$ & 0.623 \\
\hline RRQS & $70.73 \pm 12.34$ & $71.11 \pm 11.10$ & $76.86 \pm 15.40$ & 0.842 \\
\hline RRQT & $75.94 \pm 12.80$ & $75.07 \pm 11.45$ & $70.39 \pm 15.19$ & 0.624 \\
\hline BDI-II & $22.21 \pm 15.30$ & $19.64 \pm 13.40$ & $23.39 \pm 16.43$ & 0.354 \\
\hline HAM-D & $14.65 \pm 6.80$ & $13.43 \pm 7.13$ & $15.57 \pm 5.92$ & 0.228 \\
\hline $\mathrm{BAI}$ & $41.20 \pm 13.92$ & $39.29 \pm 12.77$ & $41.96 \pm 14.70$ & 0.469 \\
\hline SBQ-R & $8.97 \pm 4.75$ & $8.21 \pm 4.43$ & $9.54 \pm 4.83$ & 0.290 \\
\hline \multicolumn{5}{|c|}{ Alpha asymmetry index of FP1-FP2 } \\
\hline Low alpha band & $0.91 \pm 7.68$ & $3.17 \pm 5.01$ & $-0.81 \pm 8.55$ & 0.038 \\
\hline High alpha band & $0.48 \pm 6.32$ & $2.13 \pm 3.30$ & $-0.33 \pm 7.92$ & 0.134 \\
\hline Alpha band & $0.63 \pm 6.57$ & $2.52 \pm 3.57$ & $-0.56 \pm 7.89$ & 0.068 \\
\hline \multicolumn{5}{|c|}{ Alpha asymmetry index of F3-F4 } \\
\hline Low alpha band & $2.47 \pm 5.98$ & $5.59 \pm 4.58$ & $-0.33 \pm 5.63$ & $<0.001$ \\
\hline High alpha band & $1.54 \pm 4.83$ & $3.52 \pm 4.22$ & $0.02 \pm 5.04$ & 0.007 \\
\hline Alpha band & $1.91 \pm 4.66$ & $4.32 \pm 3.51$ & $-0.02 \pm 4.74$ & $<0.001$ \\
\hline \multicolumn{5}{|c|}{ Alpha asymmetry index of F7-F8 } \\
\hline Low alpha band & $4.96 \pm 9.29$ & $12.27 \pm 6.95$ & $-2.68 \pm 6.17$ & $<0.001$ \\
\hline High alpha band & $3.88 \pm 6.98$ & $9.56 \pm 3.76$ & $-2.01 \pm 5.37$ & $<0.001$ \\
\hline Alpha band & $4.33 \pm 7.40$ & $10.64 \pm 4.24$ & $-2.19 \pm 5.25$ & $<0.001$ \\
\hline
\end{tabular}

Values are presented as number $(\%)$ or mean \pm standard deviation.

LD group, left-dominant group; RD group, right-dominant group; CFI, Cognitive Flexibility Inventory; RRQS, Rumination Reflection Questionnaire-State; RRQT, Rumination Reflection Questionnaire-Trait; BDI-II, Beck Depression Inventory II; HAM-D, Hamilton Rating Scale for Depression; BAI, Beck Anxiety Inventory; SBQ-R, Suicidal Behavior Questionnaire-Revised.

Alpha asymmetry was measured at three frontal regions (i.e., FP1-FP2, F3-F4, F7-F8) for the low alpha band, the high alpha band, and the alpha band. 
(sLORETA). sLORETA is a source imaging method that is advantageous of solving the EEG inverse problem. ${ }^{54)}$ Based on the assumption that the source activation of a voxel is similar to that of the surrounding voxels, it applies an appropriate standardization of the current density. The lead field matrix was computed using a realistic head model that was segmented based on the Montreal Neurological Institute (MNI) 152 standard template, in which the three-dimensional solution space was restricted to only the cortical gray matter and hippocampus. $^{55)}$ The solution space was comprised of 6,239 voxels with $5-\mathrm{mm}$ resolution. Anatomical labels, such as the Brodmann areas, were provided with an appropriate transformation from MNI to Talairach space. ${ }^{56)}$

The ROls related to frontal alpha asymmetry were selected according to the results of previous neuroimaging and EEG source localization studies. They include the DLPFC, vmPFC, ACC, rACC, and sgACC. ${ }^{13-16)}$ In addition, we examined the OFC, which has been associated with cognitive deficits (e.g., rigidity, impulsivity, decision mak- ing) in suicidal individuals. ${ }^{57)}$ The source activities of the ROls in low, high, and total alpha frequency bands were then extracted. For further analyses, we calculated the alpha asymmetry indices for each ROI using source activities from both hemispheres.

\section{Statistical Analysis}

Spearman's correlation analysis was conducted to examine the associations between the asymmetry indices and the psychological measures. The specific method was selected because our data did not demonstrate a normal distribution. ${ }^{58)}$ In addition, a 5,000 bootstrap resampling technique was applied to address issues related to multiple comparisons. ${ }^{59)}$ Further correlation analysis was executed between the asymmetry indices and the psychological measures based on the groups created by the median split. Within those groups, the correlation between the SBQ-R and alpha asymmetry indices (i.e., low alpha asymmetry, high alpha asymmetry, and alpha asymmetry) was examined in each ROI. Considering that the majority
A

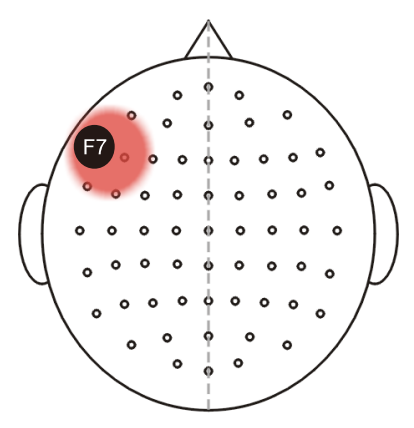

B

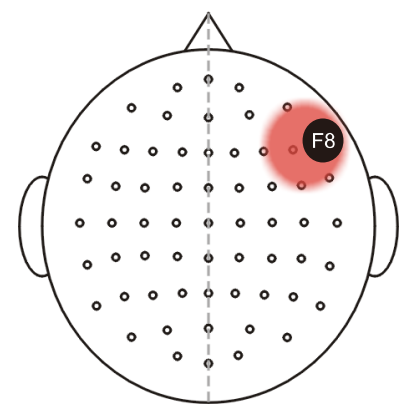

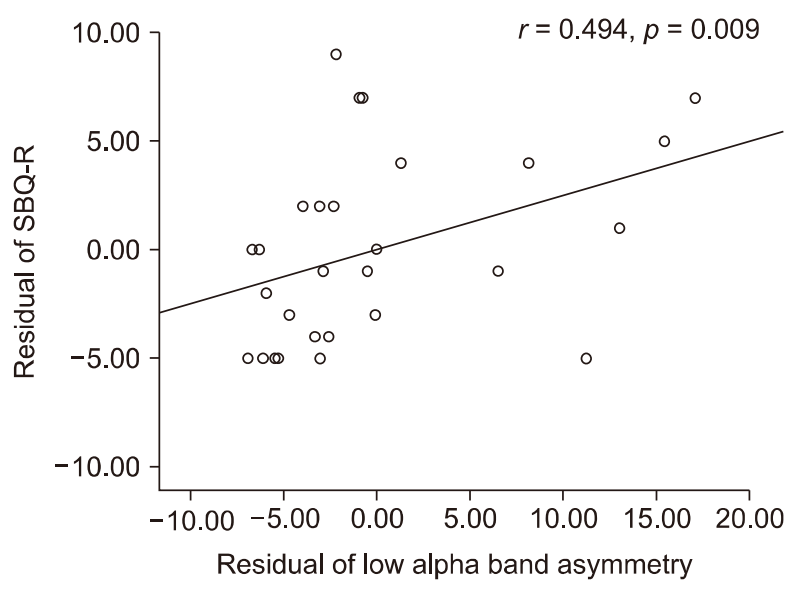

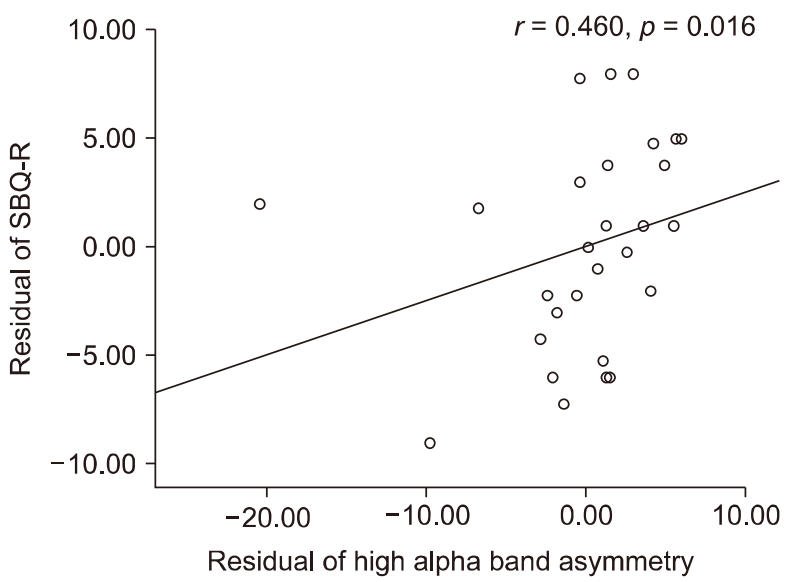

Fig. 1. Alpha asymmetry indices associated with suicidal behavior at F7-F8. In the left-dominant group (A), the asymmetry indices of the low alpha band and the alpha band were positively correlated with suicidal behavior. In the right-dominant group (B), the asymmetry index of the high alpha band was positively correlated with suicidal behavior.

SBQ-R, Suicidal Behavior Questionnaire-Revised. 
of our participants were females, sex was controlled for in all correlation analysis.

Afterwards, independent $t$ tests were performed to examine if scores on cognitive flexibility and trait and state rumination differed depending on history of prior suicide attempt and scores on the SBQ-R.

\section{RESULTS}

\section{Descriptive Statistics}

Descriptive statistics of the psychological measures and the asymmetry indices of alpha, low alpha, and high alpha in the frontal region are presented in Table 1. The mean and standard deviation for CFI, RRQS, RRQT, BDI-II, BAI, and SBQ-R were $81.36 \pm 19.04,70.73 \pm$ $12.34,75.94 \pm 12.80,22.21 \pm 15.30,41.20 \pm 13.92$, and $8.97 \pm 4.75$, respectively. The mean and the standard deviation of the asymmetry indices at each frontal site were as follows; FP1-FP2 (alpha, $0.63 \pm 6.57$; low alpha, 0.91 \pm 7.68; high alpha, $0.48 \pm 6.32$ ), F3-F4 (alpha, $1.91 \pm$ 4.66; low alpha, $2.47 \pm 5.98$; high alpha, $1.54 \pm 4.83)$, F7-F8 (alpha, $4.33 \pm 7.40$; low alpha, $4.96 \pm 9.29$; high alpha, $3.88 \pm 6.98$ ). The asymmetry indices were indicative of a higher relative alpha activity in the left hemisphere, which aligns with previous findings in patients with depression.

\section{Sensory Level Correlations}

Correlations between asymmetry indices and suicidal behavior were examined based on the groups that were created with the median scores of the asymmetry indices. No correlations were found at FP1-FP2 and F3-F4.
Table 2. Correlations coefficients between suicidal behavior and alpha asymmetry at cortical regions of interest $(n=56)$

\begin{tabular}{|c|c|c|c|c|}
\hline \multirow{2}{*}{ Variable } & \multicolumn{2}{|c|}{ LD group $(n=28)$} & \multicolumn{2}{|c|}{$R D$ group $(n=28)$} \\
\hline & SBQ-R & $p$ value & SBQ-R & $p$ value \\
\hline Past suicide attempt & $-0.591^{* *}$ & 0.001 & $-0.478^{*}$ & 0.010 \\
\hline \multicolumn{5}{|l|}{ Alpha asymmetry index } \\
\hline Low alpha & $0.494^{* *}$ & 0.009 & -0.026 & 0.897 \\
\hline High alpha & 0.344 & 0.079 & $0.460^{*}$ & 0.016 \\
\hline Alpha & $0.385^{*}$ & 0.047 & 0.241 & 0.226 \\
\hline \multicolumn{5}{|c|}{ Anterior cingulate cortex (ACC) } \\
\hline Low alpha asymmetry & 0.362 & 0.064 & -0.051 & 0.802 \\
\hline High alpha asymmetry & 0.317 & 0.107 & -0.116 & .565 \\
\hline Alpha asymmetry & 0.352 & 0.072 & -0.052 & .795 \\
\hline \multicolumn{5}{|l|}{ Rostral ACC } \\
\hline Low alpha asymmetry & $0.445^{*}$ & 0.020 & -0.009 & 0.963 \\
\hline High alpha asymmetry & $0.468^{*}$ & 0.014 & -0.139 & .490 \\
\hline Alpha asymmetry & $0.463^{*}$ & 0.015 & -0.043 & .830 \\
\hline \multicolumn{5}{|l|}{ Subgenual ACC } \\
\hline Low alpha asymmetry & 0.290 & 0.143 & -0.080 & 0.691 \\
\hline High alpha asymmetry & 0.172 & 0.392 & 0.181 & 0.367 \\
\hline Alpha asymmetry & 0.194 & 0.331 & 0.049 & 0.807 \\
\hline \multicolumn{5}{|c|}{ Dorsolateral prefrontal cortex } \\
\hline Low alpha asymmetry & $0.508^{* *}$ & 0.007 & 0.017 & 0.935 \\
\hline High alpha asymmetry & $0.436^{*}$ & 0.023 & 0.072 & 0.722 \\
\hline Alpha asymmetry & $0.455^{*}$ & 0.017 & 0.047 & 0.818 \\
\hline \multicolumn{5}{|c|}{ Ventromedial prefrontal cortex } \\
\hline Low alpha asymmetry & $0.405^{*}$ & 0.036 & 0.104 & 0.605 \\
\hline High alpha asymmetry & 0.255 & 0.200 & 0.144 & 0.475 \\
\hline Alpha asymmetry & 0.343 & 0.080 & 0.110 & 0.583 \\
\hline \multicolumn{5}{|l|}{ Orbitofrontal cortex } \\
\hline Low alpha asymmetry & $0.386^{*}$ & 0.047 & 0.139 & 0.488 \\
\hline High alpha asymmetry & 0.146 & 0.467 & 0.180 & 0.370 \\
\hline Alpha asymmetry & 0.239 & 0.239 & 0.142 & 0.481 \\
\hline
\end{tabular}

Groups were divided based on the median score (4.37) of the asymmetry index of the alpha band at F7-F8.

LD group, left-dominant group; RD group, right-dominant group; SBQ-R, Suicidal Behavior Questionnaire-Revised. ${ }^{*} p<0.05,{ }^{* *} p<0.01$.

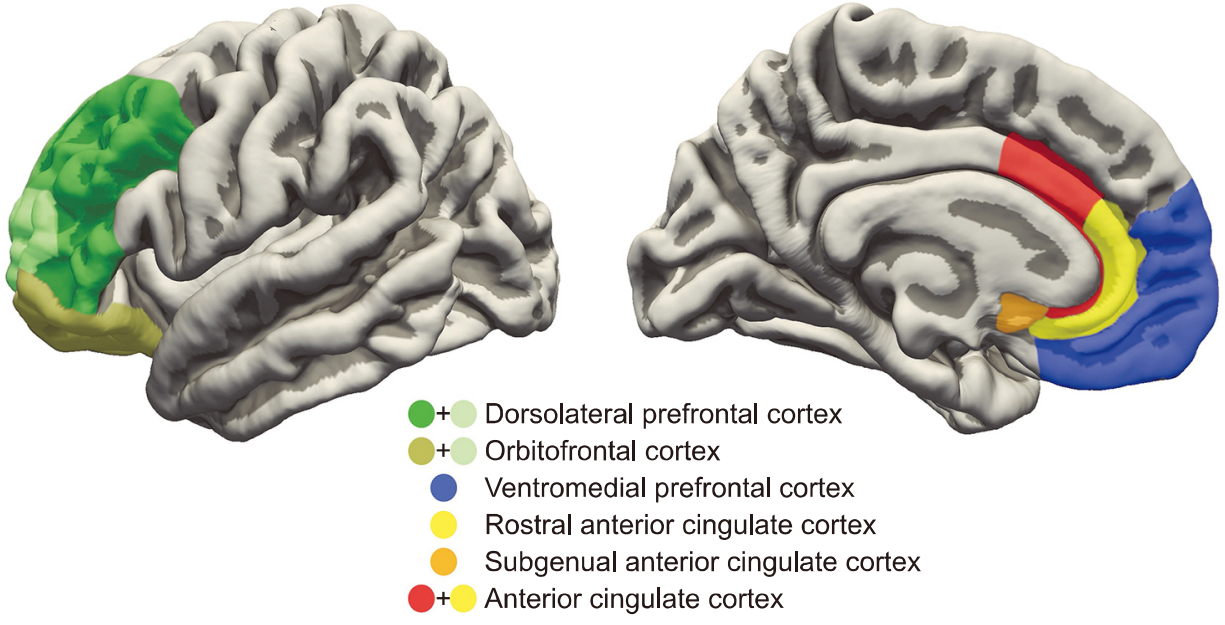

Fig. 2 Regions of interest for examination of the relationship between asymmetry of the alpha band source activity and suicidal behavior. 
Hence, these channels were disregarded from further analyses. Significant correlations were observed at F7-F8. In those who had greater alpha activity in their left frontal region, the asymmetry index of the low alpha band $(r=$ 0.494, $p=0.009)$ and that of the entire alpha band $(r=$ $0.385, p=0.047$ ) was positively correlated with suicidal behavior. In those who had greater alpha activity in their right frontal region, the asymmetry index of the high alpha band was positively correlated with suicidal behavior ( $r=$ 0.460, $p=0.016$; Fig. 1).

\section{Source Level Correlations}

ROls of source level analysis is depicted in Figure 2; correlations between suicidal behavior and the alpha asymmetry indices of each ROI in the LD and RD groups in F7-F8 are presented in Table 2. No significant correlations were found in the RD group. On the other hand, suicidal behavior was positively correlated with the asymmetry index of the low alpha band $(r=0.445, p=0.020)$, the high alpha band $(r=0.468, p=0.014)$, and the alpha band ( $r=0.463, p=0.015)$ at the rACC, as well as the asymmetry index of the low alpha band $(r=0.508, p=$ $0.007)$, the high alpha band $(r=0.436, p=0.023)$ and the alpha band $(r=0.455, p=0.017)$ at the DLPFC in the LD group. Furthermore, suicidal behavior was also positively

A

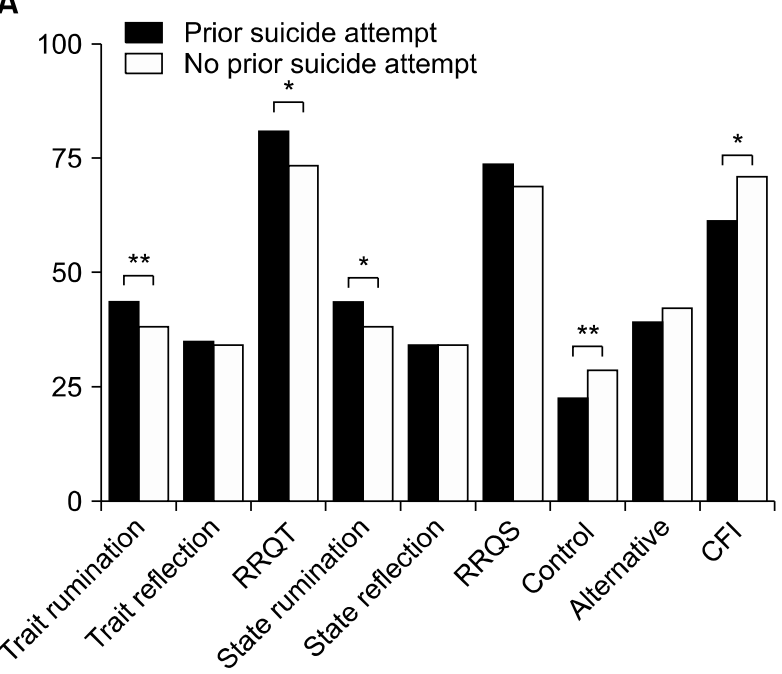

correlated with the asymmetry index of the low alpha band at the vmPFC ( $r=0.405, p=0.036)$ and the asymmetry index of the low alpha band $(r=0.386, p=0.047)$ at the OFC. There were no significant correlations between suicidal behavior and the alpha asymmetry indices at the ACC and the sgACC.

\section{T Tests}

Figure 3 shows comparisons between cognitive flexibility and ruminative tendencies and prior suicide attempts and scores on the SBQ-R. Those who had made prior suicide attempts had significantly higher scores on trait rumination $(t=-3.010, p=0.004)$, state rumination $(t=-2.496, p=0.015)$, and the overall RRQT $(t=-$ $2.390, p=0.020)$, and significantly lower scores on the control subscale of the CFI $(t=2.866, p=0.006)$ and the entire CFI ( $t=2.452, p=0.017)$ compared to those who had never attempted suicide. The difference was more pronounced when the groups in comparison were divided by their scores on the SBQ-R; those who scored higher on the SBQ-R had significantly higher scores on trait rumination $(t=-3.418, p<0.001)$, state rumination $(t=$ -3.673, $p<0.001)$, overall RRQT $(t=-2.543, p=$ $0.013)$, and RRQS $(t=-2.264, p=0.027)$, and significantly lower scores on the control subscale $(t=3.673$,

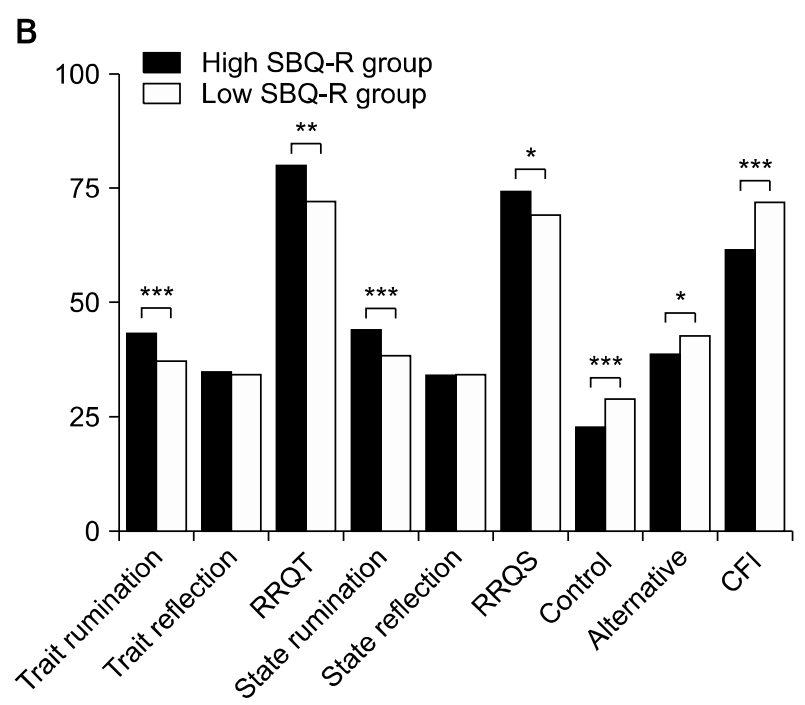

Fig. 3. Rumination and cognitive flexibility scores by group. (A) Comparisons are made between individuals with and without a history of prior suicide attempt. (B) Comparisons are made between individuals who scored high and individuals who scored low on the Suicidal Behavior Questionnaire-Revised (i.e., SBQ-R).

RRQT, Rumination Reflection Questionnaire-Trait; RRQS, Rumination Reflection Questionnaire-State; CFI, Cognitive Flexibility Scale; Control, control subscale of $\mathrm{CFI}$; Alternative, alternative subscale of CFI.

${ }^{*} p<0.05,{ }^{* *} p<0.01,{ }^{* * *} p<0.001$. 
$p<0.001)$, the alternative subscale $(t=2.193, p=0.032)$ and the entire CFI $(t=3.502, p<0.001)$ than those who scored lower on the SBQ-R.

\section{DISCUSSION}

While attempts to identify biological correlates of suicidal behaviors have increased ${ }^{60,611}$ the primary objective of our study was to examine the association between alpha asymmetry and suicidal behavior. The main findings are as follows: 1) sensory level analysis revealed a significant positive correlation between the alpha asymmetry indices of the low alpha band, the alpha band and suicidal behavior in the LD group, and a significant positive correlation between the alpha asymmetry index of the high alpha band and suicidal behavior in the RD group; 2) source level analysis revealed significant positive correlations between indices of alpha asymmetry and suicidal behavior in the LD group but not in the RD group; and 3) suicidal individuals scored higher on measures of rumination and inflexible cognitive style than non-suicidal individuals.

In the LD group, the alpha asymmetry indices of the low alpha band and the alpha band were positively correlated with suicidal behavior. In contrast, the alpha asymmetry index of the high alpha band was positively correlated with suicidal behavior in the RD group. In our study, the LD group showed greater relative alpha power in the left frontal hemisphere than in the right, which is thought to be normative in depression. ${ }^{9,62)}$ According to Graae et al., ${ }^{29)}$ adolescents with depression who had previously attempted suicide showed a greater alpha in their left frontal hemisphere than in their right. Our study has replicated the findings in adults with MDD.

Previous research has suggested that the desynchronization of the lower frequency alpha band is associated with attention. ${ }^{34,63)}$ That is, suppression of the lower frequency alpha band occurs as attentional demands increase. Suicidal individuals often experience difficulty in controlling attention (i.e., deciding which stimuli to focus on) ${ }^{32)}$ or have attentional biases toward suicide-related stimuli. ${ }^{30)}$ In this case, alterations of the lower frequency alpha band may provide profound insight into suicidal behaviors of patients with depression. Little to no research has been done to examine the specific functions of the high alpha band and its relations to suicidal behav- ior in patients with depression. To our knowledge, the only prior studies of the high alpha band reported that it may be related to semantic or task specific effects. ${ }^{34,35)}$ Therefore, future investigations of the high alpha band are warranted.

Our results revealed significant associations between the alpha asymmetry indices of the DLPFC, vmPFC, rACC, and OFC and suicidal behaviors in the LD group, but not in the RD group. The most consistent relationships were observed between the alpha asymmetry indices of the low alpha band and suicidal behavior. These findings indicate that suicide risk increases in coherence with the relative power of the lower frequency alpha band in the left hemispheres of each region. In particular, reduced activity in the left DLPFC has been shown to be associated with a tendency to negatively interpret emotional cues in MDD patients. According to Disner et al., ${ }^{64)}$ negative emotional interpretation could be a byproduct of ruminative tendencies that are reflected by hypoactivation of the left DLPFC. The study also claimed that abnormal cortical activity of the rACC might lead to ruminative tendencies in individuals with depression by inhibiting positive stimuli and simultaneously impeding the inhibition of negative stimuli. Moreover, significant reductions in gray matter in the left DLPFC and the rACC have been identified in suicidal individuals, ${ }^{65,66)}$ as has cortical thickness in the left DLPFC and the ACC. ${ }^{67)}$ These results suggest that such regions may play a significant role in suicidal behavior. In another study, Jollant et al. ${ }^{68)}$ found reduced activity in the left OFC when individuals, who had previously attempted suicide, performed risky tasks. They suggested that reduced activity of the left OFC may be related with impaired decision making. In addition, deficits in the OFC have been associated with greater impulsivity, ${ }^{25,26)}$ which is thought to indirectly increase suicide risk. ${ }^{69)}$

The reason that a relationship between alpha asymmetry of the cortical ROls and suicidal behavior was only seen in the LD group remains ambiguous. A possible explanation is that the RD group might represent individuals with atypical depression, which has discrete biological foundations. ${ }^{70)}$ It would thus be worthwhile for future studies to examine differences in alpha asymmetry among various subtypes of depression.

Lastly, we found that suicidal patients with depression were more likely to have ruminative tendencies and cognitive inflexibility than non-suicidal patients with 
depression. That is, suicidal individuals tend to fixate in their suicidal thoughts and are often unable to think of alternative solutions during mental sufferings. Such results are consistent with previous findings of cognitive rigidity in suicidal individuals. ${ }^{71,72)}$

Despite the significant findings, there are some limitations to our study. The main limitation is that we did not control for comorbidities, which may affect alpha asymmetry. For example, it is thought that the pattern of alpha asymmetry in patients with anxiety is the opposite of that of patients with MDD, in that the relative alpha power is greater in the right hemisphere than in the left. ${ }^{73)}$ Another limitation is that although we believe that the alpha asymmetry indices might reflect the cognitive rigidity of suicidal individuals, the direct relationship was not examined in our study. Future studies ought to focus on identifying the association between source level alpha asymmetry indices and cognitive abilities in MDD patients with suicidal tendencies using more sophisticated designs.

Nevertheless, our study is one of only a few that has directly examined the relationship between frontal alpha asymmetry and suicidal behavior. Moreover, to our knowledge, our study is the first to explore the relationship between source level alpha asymmetry in various cortical areas and suicidal behavior. Our results indicate that individuals with depression and suicidal behaviors exhibit an increased relative alpha power in the left hemisphere of the DLPFC, vmPFC, rACC, and OFC, which may be reflective of ruminative tendencies and cognitive rigidity. Taken together, our results suggest that alpha asymmetry, especially that of the low alpha band, may be a plausible biomarker of suicide risk.

\section{- Acknowledgments}

This work was supported by the 2017 creative research program of Inje University, and a grant from the Korea Science and Engineering Foundation (KOSEF), funded by the Korean government (NRF-2018R1A2A2A05018505).

This paper was presented at the 2017 Korean Neuropsychiatric Association Annual Autumn Meeting, Gyeongju, South Korea (October 20-21, 2017) and at the 2018 Korean Academy of Anxiety and Mood Annual Spring Meeting, Seoul, South Korea (May 25, 2018).

\section{Conflicts of Interest}

No potential conflict of interest relevant to this article was reported.

\section{REFERENCES}

1. Chesney E, Goodwin GM, Fazel S. Risks of all-cause and suicide mortality in mental disorders: a meta-review. World Psychiatry 2014;13:153-160.

2. Rihmer Z. Suicide risk in mood disorders. Curr Opin Psychiatry 2007;20:17-22.

3. Nock MK, Hwang I, Sampson NA, Kessler RC. Mental disorders, comorbidity and suicidal behavior: results from the National Comorbidity Survey Replication. Mol Psychiatry 2010;15:868-876.

4. Shim M, Im CH, Kim YW, Lee SH. Altered cortical functional network in major depressive disorder: a resting-state electroencephalogram study. Neuroimage Clin 2018;19:1000-1007.

5. Baik SY, Kim C, Kim S, Yook DW, Kim HS, Chang H, et al. The moderating effect of heart rate variability on the relationship between alpha asymmetry and depressive symptoms. Heliyon 2019;5:e01290.

6. Harmon-Jones E, Allen JJ. Behavioral activation sensitivity and resting frontal EEG asymmetry: covariation of putative indicators related to risk for mood disorders. J Abnorm Psychol 1997;106:159-163.

7. Allen JJ, lacono WG, Depue RA, Arbisi P. Regional electroencephalographic asymmetries in bipolar seasonal affective disorder before and after exposure to bright light. Biol Psychiatry 1993;33:642-646.

8. Henriques JB, Davidson RJ. Regional brain electrical asymmetries discriminate between previously depressed and healthy control subjects. J Abnorm Psychol 1990;99:22-31.

9. Henriques JB, Davidson RJ. Left frontal hypoactivation in depression. J Abnorm Psychol 1991; 100:535-545.

10. Debener S, Beauducel A, Nessler D, Brocke B, Heilemann H, Kayser J. Is resting anterior EEG alpha asymmetry a trait marker for depression? Findings for healthy adults and clinically depressed patients. Neuropsychobiology 2000;41:31-37.

11. Gotlib IH. EEG alpha asymmetry, depression, and cognitive functioning. Cogn Emot 1998;12:449-478.

12. Lindsley DB, Wicke JD. The electroencephalogram: autonomous electrical activity in man and animals. In: Thompson RF, Patterson MM, editors. Bioelectric recording techniques. New York:Academic Press; 1974. p.3-83.

13. Arns M, Bruder G, Hegerl U, Spooner C, Palmer DM, Etkin A, et al. EEG alpha asymmetry as a gender-specific predictor of outcome to acute treatment with different antidepressant medications in the randomized iSPOT-D study. Clin Neurophysiol 2016;127:509-519.

14. Escolano C, Navarro-Gil M, Garcia-Campayo J, Congedo M, De Ridder D, Minguez J. A controlled study on the cognitive 
effect of alpha neurofeedback training in patients with major depressive disorder. Front Behav Neurosci 2014;8:296.

15. Koo PC, Thome J, Berger C, Foley P, Hoeppner J. Current source density analysis of resting state EEG in depression: a review. I Neural Transm (Vienna) 2017;124(Suppl 1):109118.

16. Meyer T, Smeets T, Giesbrecht T, Quaedflieg CW, Smulders $\mathrm{FT}$, Meijer $\mathrm{EH}$, et al. The role of frontal EEG asymmetry in post-traumatic stress disorder. Biol Psychol 2015;108:62-77.

17. Koenigs M, Grafman J. The functional neuroanatomy of depression: distinct roles for ventromedial and dorsolateral prefrontal cortex. Behav Brain Res 2009;201:239-243.

18. Zald DH, Mattson DL, Pardo JV. Brain activity in ventromedial prefrontal cortex correlates with individual differences in negative affect. Proc Natl Acad Sci U S A 2002;99:2450-2454.

19. Bench CJ, Friston KJ, Brown RG, Frackowiak RS, Dolan RJ. Regional cerebral blood flow in depression measured by positron emission tomography: the relationship with clinical dimensions. Psychol Med 1993;23:579-590.

20. Mayberg HS. Frontal lobe dysfunction in secondary depression. I Neuropsychiatry Clin Neurosci 1994;6:428442.

21. Rubin KH, Chen X, McDougall P, Bowker A, McKinnon J. The Waterloo Longitudinal Project: predicting internalizing and externalizing problems in adolescence. Dev Psychopathol 1995; 7:751-764.

22. Buchsbaum MS, Wu J, Siegel BV, Hackett E, Trenary M, Abel $\mathrm{L}$, et al. Effect of sertraline on regional metabolic rate in patients with affective disorder. Biol Psychiatry 1997;41:15-22.

23. Drevets WC, Price JL, Simpson JR Jr, Todd RD, Reich T, Vannier M, et al. Subgenual prefrontal cortex abnormalities in mood disorders. Nature 1997;386:824-827.

24. George MS, Wassermann EM, Kimbrell TA, Little JT, Williams $\mathrm{WE}$, Danielson AL, et al. Mood improvement following daily left prefrontal repetitive transcranial magnetic stimulation in patients with depression: a placebo-controlled crossover trial. Am J Psychiatry 1997;154:1752-1756.

25. Best M, Williams JM, Coccaro EF. Evidence for a dysfunctional prefrontal circuit in patients with an impulsive aggressive disorder. Proc Natl Acad Sci U S A 2002;99:8448-8453.

26. Dougherty DM, Mathias CW, Marsh DM, Papageorgiou TD, Swann AC, Moeller FG. Laboratory measured behavioral impulsivity relates to suicide attempt history. Suicide Life Threat Behav 2004:34:374-385.

27. Matsuo K, Nicoletti M, Nemoto K, Hatch JP, Peluso MA, Nery FG, et al. A voxel-based morphometry study of frontal gray matter correlates of impulsivity. Hum Brain Mapp 2009;30: 1188-1195.

28. Monkul ES, Hatch JP, Nicoletti MA, Spence S, Brambilla P, Lacerda $\mathrm{AL}$, et al. Fronto-limbic brain structures in suicidal and non-suicidal female patients with major depressive disorder. Mol Psychiatry 2007; 12:360-366.

29. Graae F, Tenke C, Bruder G, Rotheram MJ, Piacentini J,
Castro-Blanco D, et al. Abnormality of EEG alpha asymmetry in female adolescent suicide attempters. Biol Psychiatry 1996;40:706-713.

30. Cha CB, Najmi S, Park JM, Finn CT, Nock MK. Attentional bias toward suicide-related stimuli predicts suicidal behavior. I Abnorm Psychol 2010;119:616-622.

31. Jollant F, Lawrence NL, Olié E, Guillaume S, Courtet P. The suicidal mind and brain: a review of neuropsychological and neuroimaging studies. World J Biol Psychiatry 2011;12:319339.

32. Keilp JG, Gorlyn M, Russell M, Oquendo MA, Burke AK, Harkavy-Friedman J, et al. Neuropsychological function and suicidal behavior: attention control, memory and executive dysfunction in suicide attempt. Psychol Med 2013;43:539551.

33. Pu S, Nakagome K, Yamada T, Yokoyama K, Matsumura H, Yamada S, et al. Suicidal ideation is associated with reduced prefrontal activation during a verbal fluency task in patients with major depressive disorder. J Affect Disord 2015;181:917.

34. Klimesch W. EEG alpha and theta oscillations reflect cognitive and memory performance: a review and analysis. Brain Res Rev 1999;29:169-195.

35. Pfurtscheller G, Neuper C, Krausz G. Functional dissociation of lower and upper frequency mu rhythms in relation to voluntary limb movement. Clin Neurophysiol 2000;111:18731879.

36. Fink A, Grabner RH, Neuper C, Neubauer AC. EEG alpha band dissociation with increasing task demands. Brain Res Cogn Brain Res 2005;24:252-259.

37. Hale TS, Smalley SL, Hanada G, Macion J, McCracken JT, McGough JJ, et al. Atypical alpha asymmetry in adults with ADHD. Neuropsychologia 2009;47:2082-2088.

38. Keilp JG, Gorlyn M, Oquendo MA, Burke AK, Mann JJ. Attention deficit in depressed suicide attempters. Psychiatry Res 2008;159:7-17.

39. Surrence K, Miranda R, Marroquín BM, Chan S. Brooding and reflective rumination among suicide attempters: cognitive vulnerability to suicidal ideation. Behav Res Ther 2009;47: 803-808.

40. Glenn CR, Kleiman EM, Coppersmith DDL, Santee AC, Esposito EC, Cha CB, et al. Implicit identification with death predicts change in suicide ideation during psychiatric treatment in adolescents. I Child Psychol Psychiatry 2017;58: 1319-1329.

41. Nock MK, Park JM, Finn CT, Deliberto TL, Dour HJ, Banaji MR. Measuring the suicidal mind: implicit cognition predicts suicidal behavior. Psychol Sci 2010;21:511-517.

42. Heo S. The role of cognitive flexibility in the relationship between perfectionism and psychological maladjustment. Seoul:Seoul National University;2011. [Dissertation].

43. Park SH. Development of the mindfulness scale. Bucheon: The Catholic University of Korea;2006. [Dissertation]. 
44. Lee Y, Song J. A study of the reliability and the validity of the BDI, SDS, and MMPI-D scales. Korean J Clin Psychol 1991;10:98-113.

45. Kwon SM. Differential roles of dysfunctional attitudes and automatic thoughts in depression: An integrated cognitive model of depression. St. Lucia:University of Queensland;1992. [Dissertation].

46. Lee HS. The development and outcome evaluation of the Korean military suicide prevention program based on the community whole-system approach. Seoul:Korea University; 2009. [Dissertation].

47. Osman A, Bagge CL, Gutierrez PM, Konick LC, Kopper BA, Barrios FX. The Suicidal Behaviors Questionnaire-Revised (SBQ-R): validation with clinical and nonclinical samples. Assessment 2001;8:443-454.

48. Batterham PJ, Ftanou M, Pirkis J, Brewer JL, Mackinnon AJ, Beautrais A, et al. A systematic review and evaluation of measures for suicidal ideation and behaviors in population-based research. Psychol Assess 2015;27:501-512.

49. Lee H, Kwon J. Validation for the Beck Scale for Suicide Ideation with Korean university students. Korean I Clin Psychol 2009;28:1155-1172.

50. Dekker MK, Sitskoorn MM, Denissen AJ, van Boxtel GJ. The time-course of alpha neurofeedback training effects in healthy participants. Biol Psychol 2014;95:70-73.

51. Carvalho A, Moraes $H$, Silveira $H$, Ribeiro P, Piedade RA, Deslandes AC, et al. EEG frontal asymmetry in the depressed and remitted elderly: is it related to the trait or to the state of depression? J Affect Disord 2011;129:143-148.

52. Gold C, Fachner J, Erkkilä J. Validity and reliability of electroencephalographic frontal alpha asymmetry and frontal midline theta as biomarkers for depression. Scand I Psychol 2013;54:118-126.

53. Jacobs GD, Snyder D. Frontal brain asymmetry predicts affective style in men. Behav Neurosci 1996;110:3-6.

54. Pascual-Marqui RD. Standardized low-resolution brain electromagnetic tomography (sLORETA): technical details. Methods Find Exp Clin Pharmacol 2002;24 Supp/ D:5-12.

55. Fuchs M, Kastner J, Wagner M, Hawes S, Ebersole JS. A standardized boundary element method volume conductor model. Clin Neurophysiol 2002;113:702-712.

56. Brett M, Johnsrude IS, Owen AM. The problem of functional localization in the human brain. Nat Rev Neurosci 2002;3: 243-249.

57. LeGris J, van Reekum R. The neuropsychological correlates of borderline personality disorder and suicidal behaviour. Can J Psychiatry 2006;51:131-142.

58. Gibbons JD, Chakraborti S. Nonparametric statistical inference. In: Lovric M, editor. International encyclopedia of statistical science. Berlin, Heidelberg:Springer;2011. p.977-979.

59. Westfall PH. On using the bootstrap for multiple comparisons. J Biopharm Stat 2011;21:1187-1205.
60. Atescelik M, Yilmaz M, Korkmaz S, Goktekin MC, Gurger M, Ilhan $\mathrm{N}$. The relationship between ghrelin and copeptin levels, and anxiety and depression levels in suicide attempts. Clin Psychopharmaco/ Neurosci 2017;15:256-260.

61. De Berardis D, Olivieri L, Rapini G, Di Natale S, Serroni N, Fornaro $\mathrm{M}$, et al. Alexithymia, suicide ideation and homocysteine levels in drug naïve patients with major depression: a study in the "real world" clinical practice. Clin Psychopharmacol Neurosci 2019;17:318-322.

62. Deslandes AC, de Moraes H, Pompeu FA, Ribeiro P, Cagy M, Capitão C, et al. Electroencephalographic frontal asymmetry and depressive symptoms in the elderly. Biol Psychol 2008; 79:317-322.

63. Klimesch W, Doppelmayr M, Russegger H, Pachinger T, Schwaiger J. Induced alpha band power changes in the human EEG and attention. Neurosci Lett 1998;244:73-76.

64. Disner SG, Beevers CG, Haigh EA, Beck AT. Neural mechanisms of the cognitive model of depression. Nat Rev Neurosci 2011;12:467-477.

65. Liu H, Wang Y, Liu W, Wei D, Yang J, Du X, et al. Neuroanatomical correlates of attitudes toward suicide in a large healthy sample: a voxel-based morphometric analysis. Neuropsychologia 2016;80:185-193.

66. Wagner G, Koch K, Schachtzabel C, Schultz CC, Sauer H, Schlösser RG. Structural brain alterations in patients with major depressive disorder and high risk for suicide: evidence for a distinct neurobiological entity? Neuroimage 2011;54:16071614.

67. Wagner G, Schultz CC, Koch K, Schachtzabel C, Sauer H, Schlösser RG. Prefrontal cortical thickness in depressed patients with high-risk for suicidal behavior. I Psychiatr Res 2012:46:1449-1455.

68. Jollant F, Lawrence NS, Olie E, O'Daly O, Malafosse A, Courtet $\mathrm{P}$, et al. Decreased activation of lateral orbitofrontal cortex during risky choices under uncertainty is associated with disadvantageous decision-making and suicidal behavior. Neuroimage 2010;51:1275-1281.

69. Bender TW, Gordon KH, Bresin K, Joiner TE Jr. Impulsivity and suicidality: the mediating role of painful and provocative experiences. J Affect Disord 2011;129:301-307.

70. Pandey R, Gupta G. Hemispheric asymmetry in depression: an overview. Indian J Soc Sci Res 2009;6:16-28.

71. Miranda R, Gallagher M, Bauchner B, Vaysman R, Marroquín B. Cognitive inflexibility as a prospective predictor of suicidal ideation among young adults with a suicide attempt history. Depress Anxiety 2012;29:180-186.

72. Prezant DW, Neimeyer RA. Cognitive predictors of depression and suicide ideation. Suicide Life Threat Behav 1988;18: 259-264.

73. Fingelkurts AA, Fingelkurts AA. Altered structure of dynamic electroencephalogram oscillatory pattern in major depression. Biol Psychiatry 2015;77:1050-1060. 